interesting mechanisms giving rise to this property are conceivable. For instance, the concept of supramolecular springs could, in principle, be used in conjunction with other known mechanisms for NLC to enhance the effect even further. These developments and discoveries relating to systems exhibiting such highly anomalous yet potentially useful macroscopic properties are greatly encouraging and may pave the way to a new generation of advanced functional materials with exceptional properties.

Ruben Gatt, Roberto Caruana-Gauci and Joseph N. Grima are at the Metamaterials Unit, Faculty of Science, University of Malta, Msida MSD 2080, Malta.

e-mail: joseph.grima@um.edu.mt

References

1. Lakes, R. S. \& Wojciechowski, K. W. Phys. Status Solidi B $245,545-551$ (2008)
2. Cairns, A. B. et al. Nature Mater. 12, 212-216 (2013)

3. Baughman, R. H., Stafstrom, S., Cui, C. \& Dantas, S. Science 279, 1522-1524 (1998).

4. Fortes, A. D., Suard, E. \& Knight, K. S. Science 331, 742-746 (2011)

Grima, J. N., Attard, D., Caruana-Gauci, R. \& Gatt, R. Scripta Materialia 65, 565-568 (2011).

6. Gatt, R. \& Grima, J. N. Phys. Status Solidi Rapid Res. Lett. 2, 236-238 (2008)

7. Barnes, D. L., Miller, W., Evans, K. E. \& Marmier, A. S. H. Mech. Mater. 46, 123-128 (2012).

8. Nicolaou, Z G. \& Motter, A. E. Nature Mater. 11, 608-613 (2012).

9. Evans, K. E., Nkansah, M. A., Hutchinson, I. \& Rogers, S. Nature 353, 124-124 (1991)

10. Evans, J. S. O. J. Chem. Soc. Dalton Trans. 3317-3326 (1999).

\title{
LIVING CRYSTALS
}

Aggregation phenomena have long held the promise of bridging the organic and inorganic realms. Crystallization a variant of that category - has traditionally displayed a triumph of the ordering tendency over the disruptive influences of diffusive randomness and thermal motion, giving rise to periodic arrangements of the coalescing particles. But electrodeposition and diffusionlimited aggregation show that crystal growth under far-from-equilibrium conditions can generate structures with branched, disorderly and fractal forms, familiar from the living world.

All the same, that result is a static one. When, however, aggregating particles possess their own autonomous means of directional motion, selforganization does not necessarily freeze out a particular arrangement but exhibits dynamical forms. At first glance, these could seem to be uniquely biological - the swarming of fish and birds, the swirling and channelling of bacteria and slime moulds - for inorganic particles (robots aside) don't generally have their own means of propulsion. Not, that is, until the past decade.

The discovery of self-propelled platinum-gold nanorods by Thomas Mallouk, Ayusman Sen and their coworkers ${ }^{1}$, for instance, has stimulated the development of a whole range of microscopic entities that will move through solution or on surfaces driven by surface-tension gradients, gas evolution or other forces ${ }^{2-5}$. In Mallouk and Sen's work, such gradients were created and sustained by catalytic decomposition of hydrogen peroxide to oxygen. It is now possible to recognize 'active matter' as a new category of material that encompasses everything from Escherichia coli to the cytoskeleton, catalytic nanoparticles and microporous gels 6 .

Synthetic micro- and nanostructures of this sort can, in isolation, be regarded as potential 'nanomotors'. But when present in concentrated suspensions, they may interact with one another both phoretically and hydrodynamically to give rise to forms of collective behaviour reminiscent of flocking and swarming. This makes such active matter a potential model system for exploring macroscopic biological self-organization - but it also seems we must expect novel behaviour to manifest, such as aggregation into clusters of preferred average sizes ${ }^{7}$.

Palacci et al. now report a striking new variation on this theme: colloidal self-propelled particles that can gather into 'living crystals', which are constantly forming, growing and fragmenting ${ }^{8}$. What is more, the propulsion can be easily switched on and off using light.

These particles are cubes of haematite about a micrometre across, encased asymmetrically in polymer shells so as to expose a small part of the cube. When suspended in basic hydrogen peroxide solution and illuminated with blue-violet light, the haematite decomposes the peroxide and leads to a chemical gradient. That in turn induces osmotic flow close to a wall or surface, which, thanks to chance asymmetries, pumps the particles towards it.

This 'diffusiophoretic' motion also generates an effective attraction between particles close to the surface, resulting in the formation of close-packed

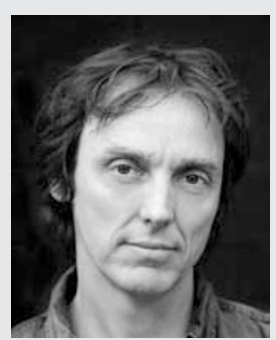

PHILIP BALL

two-dimensional clusters at the container walls, with an average size of 35 particles under the conditions studied. These melt by thermal diffusion once the propulsion is turned 'off', and the pseudo-two-dimensional dispersion of particles undergoes a transition in density fluctuations above a critical concentration, as predicted for other active particles ${ }^{9}$. This controlled aggregation, coupled with the possibility of magnetic steering of the haematite, could offer new directions for materials assembly and dynamic activity.

References

1. Paxton, W. F. et al. J. Am. Chem. Soc. 126, 13424-13431 (2004).

2. Golestanian, R., Liverpool, T. B. \& Ajdari, A. Phys. Rev. Lett. 94, 220801 (2005)

3. Tao, Y. G. \& Kapral, R. ChemPhysChem 10, 770-773 (2009).

4. Sharma, R., Chang, S. T. \& Velev, O. D. Langmuir 28, 10128-10135 (2012).

5. Gao, W., Uygun, A. \& Wang, J. J. Am. Chem. Soc. 134, 897-900 (2012).

6. Ramaswamy, S. Annu. Rev. Condens. Matter Phys. 1, 323-345 (2010)

7. Theurkauff, I., Cottin-Bizonne, C., Palacci, J., Ybert, C. \& Bocquet, L. Phys. Rev. Lett. 108, 268303 (2012).

8. Palacci, J., Sacanna, S., Steinberg, A. P., Pine, D. J. \& Chaikin, P. M. Sci. Express http://dx.doi.org/10.1126/ science.1230020 (2013).

9. Fily, Y. \& Marchetti, M. C. Phys. Rev. Lett. 108, 235702 (2012). 